\title{
L'importance de la grammaire créole dans la mise en place d'un enseignement de LVR : Les modalités du futur et du conditionnel en créole guadeloupéen
}

\author{
Juliette FACTHUM-SAINTON \\ Université des Antilles et de la Guyane-CRREF, EA 4538
}

\section{Résumé}

La présente description du futur en ké, et du conditionnel en té ké est abordée sous un angle triple : en synchronie, en diachronie et selon une approche contrastive créole-français, afin de répondre à la demande d'enseignants en Guadeloupe. L'approche descriptive est pragmatique. Dans un souci didactique, ces modalités sont présentées au sein de la combinaison tékéjaka, point de saturation du groupe TMA en créole guadeloupéen.

\section{Mots-clés}

Modalités du futur ké, modalité du conditionnel té ké, le groupe TMA, le noyau prédicatif.

\begin{abstract}
The present description of the future marked ké, and of the conditional marked té ké, provides a triple approach: a diachronic one, a synchronic one and a contrastive approach, in order to answer the numerous teachers' demands in Guadeloupe. The description answers a pragmatic requirement. The didactic perspective leads us to present those two modalities within the dynamics combining tékéjaka, the saturation point in Guadeloupean Creole.
\end{abstract}

\section{Keywords}

Future as a modality, the conditional as a modality, the TMA as a constituent, the predicative nucleus. 


\section{Pourquoi une présentation en synchronie, en diachronie et un traitement de la grammaire créole en didactique contextuelle?}

Aujourd'hui, la question de la maîtrise de la grammaire créole ne se pose pas dans les mêmes termes qu'elle s'est posée au milieu des années soixante-dix. Il y a plus de trente ans, le défi était de produire une grammaire des formes créoles les plus déviantes de la norme française, ainsi légitimant la revendication identitaire.

Depuis l'entrée officielle du créole à l'école en Guadeloupe, 2001, les tendances ont basculé, à l'insu des créolophyles : on découvre que le créole est pratiqué à l'école au cœur d'un mélange de langues; on reconnaît qu'il en est de même pour le français. L'enjeu de la grammaire du créole est celui d'une approche contrastive créole-français, afin que l'élève apprenne à faire la différence entre les formes des deux langues, et que l'enseignant ait des points de repère sur les contraintes potentielles à l'apprentissage des langues en milieu créole.

L'approche en synchronie consiste en l'étude de la langue à un moment donné, par exemple l'arrêt sur images du créole, des années soixante à nos jours, ce qui est une nécessité ; mais il est souhaitable pour l'école, qu'une telle étude se réalise en empruntant son approche à la méthode contrastive, mettant ainsi vis-à-vis le créole et le français.

Le traitement d'une langue en diachronie consiste en l'étude de la langue dans son évolution au cours du temps. Toute approche descriptive du créole en synchronie, dans les domaines de la phonologie, de la morphologie, et de la syntaxe, ne peut se passer de l'éclairage de la diachronie, le créole, né en grande partie de l'autonomisation des registres populaires de français, ne s'étant pas tout à fait sevré de la langue-mère. Les processus de mise en place des mots qui vont servir à forger les catégories grammaticales du créole au XVIIIe siècle, les évolutions au sein de ces catégories, plus ou moins présentes aujourd'hui, constituent des points d'ancrage important pour un enseignant de LVR.

Cette vision en diachronie ne peut se passer d'une mise en perspective de l'évolution des autres créoles des Antilles : les textes les plus anciens de la littérature créole, ceux du XVIIIe siècle, laissent entrevoir qu'il est fiable d'émettre l'hypothèse qu'aux origines, il y avait des pratiques grammaticales indifférenciées entre les créoles insulaires. Se plonger, ne serait-ce que furtivement dans l'évolution qui mène à cette différenciation, est de nature à permettre à l'enseignant de LVR de faire le point et de se retrouver.

\section{Eclairer la notion de modalité. Revisiter la terminologie ${ }^{1}$}

Avant tout propos, il importe d'éclairer le concept derrière le terme «modalité ». Il est acquis dans certaines grammaires énonciatives, que le futur et le conditionnel ne sont pas des « temps », même si le futur apparaît sur l'axe du temps dit universel, dans l'ordre "PasséPrésent-Futur ». La tradition descriptiviste de la grammaire anglaise d'aujourd'hui est une référence en la matière (Rotgé et Lapaire, 2014). Le point d'appui d'une telle analyse est que dans un énoncé, un procès évoqué au futur ne fait pas encore partie de l'expérience tangible du locuteur-énonciateur et qu'on ignore s'il fera jamais partie du factuel. Selon cette vision, seuls le passé et le présent sont classés parmi les temps car ils expriment le factuel, le palpable. Conditionnel et futur sont classés comme modalités. Dans une telle approche, il y a

\footnotetext{
${ }^{1}$ Cet article a été réalisé avec le concours d'Olivia Boudhau, étudiante en Master 2 MEEF à l'ESPE de Guadeloupe.
} 
des phrases classées modales et des phrases rangées factuelles. Appliquant cette classification au créole, les énoncés 1 et 2 sont modaux, tandis que l'énoncé 3 est factuel :

1. An té ké manjé on ti kalalou, si an té pé (Je mangerais un bon plat de calalou, si j'en avais les moyens)

2. An ké manjé on ti kalalou dèmen, si an pé (Je mangerai un bon plat de calalou, demain, si j'en ai les moyens)

3. Alo, alo! An ka manjé on ti kalalou (Allo, allo! Je suis en train de manger un bon plat de calalou).

Mais le propos des grammairiens sur la modalité est encore plus complexe que cela. Le concept, modalité, est généralement attribué à la réflexion des logiciens. Ce fut Aristote, philosophe de la Grèce antique (384-322) qui en jeta les bases. Il semble aussi que les philosophes et théologiens de la scolastique reprirent à leur compte ce concept, entre le Xe et le XIIe siècle et que les linguistes contemporains en auraient hérité (Lapaire et Rotgé, 1991 : 370). Selon la logique aristotélicienne, la modalité dans un énoncé est une prise de position de l'énonciateur, un commentaire, par rapport à la proposition qu'il formule. Soit l'énoncé suivant en créole :

4. Gwo lapli menm, ké tonbé oswèla (De très grosses pluies tomberont plus tard).

Le locuteur énonciateur de cette phrase, après avoir scruté le ciel et les nuages conclut et fait un commentaire ou porte un jugement mettant en relation le sujet lapli et le verbal tonbé.

Selon Creissels (2004), le futur énonce une prédiction. Tout comme le futur, le conditionnel n'exprime pas une action réalisée, mais l'intention de l'auteur quant à cette action.

Il existe une notion qui sera examinée de manière pragmatique dans le paragraphe ci-dessous : c'est celle de «l'aspect». L'aspect est la manière dont l'énonciateur envisage le procès : ce dernier vient de commencer ou de se terminer sòti; est en cours de réalisation $(k a)$, est accompli (ja), n'est pas accompli (poko/pòkò), etc. Nous ne pouvons faire l'impasse sur l'aspect, car ké sera analysée dans son rapport avec le temps, té et avec les aspects ja et ka. Ce sont ces seules marques avec lesquelles ké peut se combiner.

Nous mettons en garde le lecteur contre ceci : la terminologie employée pour caractériser les aspects diffère selon les écoles et les traditions linguistiques.

Maintenant, revisitons la terminologie. Parfois, lorsqu'il est question d'une proposition principale suivie d'une proposition subordonnée, les termes suivants «potentiel » et «irréel » sont employés dans certaines traditions linguistiques, alors que dans d'autres, ce sont les terminologies «irréel du présent » et «irréel du passé » qui sont privilégiées. Ces différences terminologiques sont liées à des approches nées de la variété des écoles. Lorsqu'il sera question ci-dessous de travailler le contraste créole-français, la présente étude a fait le choix de potentiel et d'irréel.

\section{Présenter l'objet de l'étude : futur et conditionnel en créole}

Il est attesté dans les langues du monde que le futur n'a pas toujours une forme consacrée, plusieurs tournures idiomatiques, ainsi que le verbe aller au présent, pouvant être employés pour son expression. La langue anglaise illustre bien ce phénomène, mais c'est aussi un petit 
peu le cas en français ${ }^{2}$. Le créole guadeloupéen n'échappe pas à cette règle. La notion de futur, en guadeloupéen, peut être exprimée par : pou (l'idée de projet ou d'intention) ; ni pou (l'idée d'intention à laquelle s'ajoute une exigence); dwèt (l'idée de contrainte); kalé/ka'y (l'idée d'imminence, de futur proche, de futur immédiat), ké (le futur dans son expression la plus neutre). Ces marques du futur sont placées, chacune à l'exclusion de l'autre, entre le sujet et le noyau prédicatif :

5. An pou ay Trinidad (j'ai l'intention de me rendre à Trinidad)

6. Ou ni pou ou aprann lèson a'w oswèla (Tu dois apprendre tes leçons ce soir)

7. I dwèt rantré lopital dèmen menm (Il doit rentrer à l'hôpital dès demain)

8. Tansyon! zasyèt-la ka'y tonbé asi pyé a'w (Attention! l'assiette va te tomber sur le pied).

9. Dèmen an ké ni karant lanné si tèt an mwen (Demain, j’aurai quarante ans).

En plaçant té devant chacune des marques précédentes en 5,6 , 7, et 8 , on obtient d'autres formes de l'expression du futur plus ou moins marquées par le passé, phénomènes importants cependant maintenus hors de la présente perspective. En effet, ci-dessous, toutes les expressions du futur ne seront pas traitées.

Seule sera décrite, et de façon pragmatique, la marque ké représentant le futur dans son expression la plus simple et la plus neutre. Nous traiterons également de la forme du conditionnel té ké obtenue à partir du futur, mais seulement dans son interaction avec le potentiel et l'irréel :

10. Futur : An ké vini, pli ta (Je viendrai plus tard)

11. Conditionnel : Si ou té ka vini pli ta, nou té ké manjé ansanm

(Si tu venais plus tard, nous mangerions ensemble).

N.B.1 : La forme négative du futur est péké :

12. An péké vini pli ta (Je ne viendrai pas plus tard)

Remarque 1: A la forme négative du futur, il est attesté une harmonie vocalique : l'unité lexicale de la forme négative en créole $p a$ devient [pe] pé, sous l'influence de la marque ké du futur dont la forme phonique (le signifiant) se termine par la voyelle [e] é.

N.B.2 : La forme négative du conditionnel est pa té ké :

13. Nou pa té ké manjé ansanm (Nous ne mangerions pas ensemble/ nous n'aurions pas mangé ensemble)

Dans les CBLF (créoles à base lexicale française) de l'Atlantique, le futur et le conditionnel ont été diversement traités et font l'objet d'une abondante littérature, entre autres : Bernabé (1983), Damoiseau (1984, 1999, 2012), Spears (1997), Fattier (2010), G. Hazaël-Massieux (1996), Facthum-Sainton (1997), M.-C. Hazaël-Massieux (2008), Jeannot-Fourcaud (2003), DeGraff (1992, 2004), et bien d'autres. Par ailleurs, les temps, modes et aspects désormais TMA dans cette étude, ont été au cœur de grands débats sur les typologies des langues

\footnotetext{
${ }^{2}$ En anglais, le futur peut s'exprimer par les tournures idiomatiques au présent: to be going to, to be on the verge of, to be to, to be about to, ainsi que par shall/will suivi du verbe. En français, peuvent exprimer une action future, suivie d'un adverbe de temps futur, le verbe "aller"+l'infinitif, le temps présent lui-même, et enfin la flexion dite du futur ajoutée à la base verbale, cette flexion étant -erai, eras, era, erons, erez, eront pour les verbes du premier groupe, tandis que les flexions des verbes du deuxième groupe sont : irai, iras, etc. Quant aux verbes du troisième groupe, ils sont diversement mis en flexion. Là encore, cette flexion du français, telle que présentée ici, rejoint les versions traditionnelles puisque l'on peut dire que toute base verbale du français, quel que soit le groupe auquel il appartient se termine au futur par les flexions- ai, as, a, ons, ez, ont correspondant à la personne.
} 
créoles : Bickerton (1981, 1984, 1988), Arends, Muyskens et Smith (1995), Alleyne (1996), Mufwene (1985, 2001). Cette féconde littérature laisse apparaître des différences de terminologie qui sans doute cachent des différences d'appréciation entre grammairiens. Quant à moi, je désignerai par le terme "marque », les unités lexicales chargées de véhiculer les TMA en créole, té, ké, $k a$, etc. Parfois lorsque le terme «forme » s'imposera, il sera aussi utilisé.

La considération qui suit est importante: quelle que soit l'école de rattachement du descriptiviste, les grammairiens et les linguistes s'entendent sur le fait que les créoles de l'Atlantique se partagent les trois traits suivants, dans l'expression des TMA, même indépendamment de leur langue pourvoyeuse de lexique. Ces traits sont exposés ci-dessous en $\mathrm{a}, \mathrm{b}$ et $\mathrm{c}$, avec des exemples guadeloupéens. Nous nous référons en particulier à Damoiseau (2012) :

a) Les créoles expriment les temps, modes et aspects, par le biais de marques TMA placées entre le sujet grammatical et le noyau prédicatif ce dernier étant souvent une forme verbale, comme dans les exemples suivants :

14. An ké vini (Je viendrai)

15. Nou té ké manjé (Nous mangerions)

Dans l'énoncé 14, le sujet est An et le noyau prédicatif est le verbal vini. La marque TMA faisant partie du syntagme prédicatif est ké.

b) En créole, il arrive fréquemment que le noyau prédicatif appartienne à des catégories grammaticales autres que le verbe. Soit les énoncés suivants :

16. An ké kontan (Je serai content).

Dans l'énoncé An ké kontan, le noyau prédicatif est une forme adjectivale : kontan.

17. An ké anbala (Je serai là-bas). Le noyau prédicatif est un adverbe de lieu, anbala.

18. Lè an ké gran, an ké mizisyen (Quand je serai grand, je serai musicien). Dans la proposition principale an ké mizisyen, le noyau prédicatif mizisyen est un substantif (un nom).

c) Les unités lexicales TMA et le noyau prédicatif sont tous deux invariables, quelle que soit la personne. Exemples :

19. An ké travay, ou ké travay, i ké travay, nou ké travay, zòt ké travay, yo ké travay (Respectivement: je travaillerai, tu travailleras, il/elle travaillera, nous travaillerons, vous travaillerez, ils/elles travailleront).

Le même principe de nature grammaticale du noyau prédicatif s'applique à la forme du conditionnel. Le noyau prédicatif peut être un verbal, un adjectif, un adverbe ou un nom.

20. An té ké alé, si... (alé est un verbal) (J'irais si...)

21. An té ké kontan, si... (kontan est un adjectif) (Je serais content si.../j'aurais été content si...)

22. An té ké anbala, si... (anbala est un adverbe) (Je serais là-bas/ j'aurais été là-bas, si...)

23. An té ké mizisyen, si... (mizisyen est un nom) (Je serais musicien/j'aurais été musicien si...)

Remarque 2: Il est un point auquel le lecteur doit s'accrocher: en créole, la forme du conditionnel dite "présent» est la même forme que celle du conditionnel dite "passé ». Autrement dit, an té ké alé traduit aussi bien «j'irais » que «je serais allé ». Nous traiterons de ce trait, plus loin, dans les approches contrastives créole/français. 


\section{Mais quelle est l'origine de cette marque $k e ́$ ?}

Grâce à la linguistique diachronique qui regarde les éléments décrits dans leur dynamique évolutive, il est reconnu aujourd'hui que ces principales marques de TMA du créole, té, ké, ja, $k a$, poko, et bien d'autres non évoquées ici, sont issues d'un processus appelé, "grammaticalisation ». Au moment de la formation des classes grammaticales du créole, au XVIIIe siècle selon la créolistique, des mots français ou d'origine autre ont perdu leur statut grammatical originel pour endosser celui de TMA en créole. Chaudenson (1992), G. HazaëlMassieux (1996), Alleyne (1996) et M.G. Hazaël-Massieux (2008) notent ce processus de grammaticalisation dont procèdent par exemple té, ké, $k a$. Des linguistes, entre autres, Kriegel et Arends (2003), ont travaillé sur ce processus de façon plus générale, ce phénomène survenu en diachronie ayant joué un rôle de premier plan dans la mise en place des grammaires créoles.

Il est prêté à ké une origine partiellement française (G. Hazaël-Massieux, 2008 : 434-435). ké serait le résultat d'une succession de contractions ainsi exprimées en $\mathrm{a}, \mathrm{b}$ et $\mathrm{c}$ :

a) La première contraction serait celle de $k a+a l e ́$ (aller) devenant kalé, ce qui est une affirmation de l'antériorité de ka sur ké.

b) La forme kalé aurait développé une forme plus courte kaé, phonétiquement réalisée [kaj], graphie aujourd'hui $k a^{\prime} y$. Notons que kalé et $k a^{\prime} y$ vont perdurer pour exprimer, non pas le futur simple, mais le futur proche, le futur immédiat, l'imminence :

- Tansyon, zasyèt-la ka'y tonbé (Attention, l'assiette va tomber) : futur proche

- An ka'y mété manjé si difé (mot à mot: je vais mettre le repas au feu) : futur immédiat.

c) La troisième étape serait celle ou kaé se serait contracté et finalement donne ké, l'expression la plus simple du futur en créole.

M.-C. Hazaël-Massieux adhère à cette analyse de la formation du futur en créole, selon les étapes précédentes, tout en faisant référence à un autre étymon possible, en lien avec le préverbal ké du kikongo (2008: 435), sans toutefois préciser laquelle des variétés dites kikongo est visée ${ }^{3}$. Valdman, cité par l'auteur précédent, fait l'hypothèse d'une forme sortie du parler bantou de mayombe.

\section{Le futur et le conditionnel dans la distribution « Temps-Mode-Aspect »}

En créole guadeloupéen, l'ordre des marques est exclusivement Temps-Mode-Aspect, au sein d'un syntagme prédicatif quelconque, par exemple, té-ké-ka. Cependant, diverses occurrences sont possibles au sein de cette distribution.

En ce qui concerne le futur ké, les marques lui sont antéposées comme c'est le cas pour le seul marqueur té, ou postposées ; c'est le cas des marqueurs aspectuels ja et ka. Ci-dessous, le groupe TMA, mis en caractères gras, peut être composé de deux marques comme suit :

24. Mode seul : An ké kontan (Je serai content)

25. Temps-Mode : An té ké kontan (Je serais content)

26. Mode-Aspect :

a) A minui, an ké ja rivé (A minuit, je serai (déjà) arrivé)

\footnotetext{
${ }^{3}$ Hazaël-Massieux propose l'énoncé : Yá ké kitíka «Il est en train de manger la banane ». On notera ici qu'il s'agit d'une forme du présent progressif, alors qu'en créole, il s'agit du futur. Alors que dans la phrase mú ké kwénda na kitsása «je partirai à Kinshasa », le marqueur en question a une valeur de futur.
} 
b) A minui, an ké ka rivé (A minuit, je serai en train d'arriver).

27. Les autres combinaisons, avec trois marques, comme ci-dessous sont motivées par té ké:

a) Temps-Mode-Aspect : An té ké ka rivé (Je serais en train d'arriver)

b) Temps-Mode-Aspect : An té ké ja rivé (Je serais (déjà) arrivé)

Lorsque le groupe TMA est composé de 4 marques, avec deux marques aspectuelles l'une à côté de l'autre (Temps-Mode-Aspect-Aspect), c'est le point de saturation de la combinaison (J. Sainton, 1997)

c) An té ké ja ka rivé (Je serais déjà en train d'arriver). Ké Ja exprime alors l'accompli ja dans le futur.

(L'aspect itératif est attesté dans l'expression du futur :

$\underline{\text { Remarque } 3}$ : Pour que $k a$ soit attesté à la droite de $k e ́$, comme dans ces exemples ci-dessus, $26 \mathrm{~b}$ et $27 \mathrm{a}$, il faut et il suffit que le noyau prédicatif corresponde exclusivement à une seule catégorie grammaticale, celle du verbe ${ }^{4}$. Les exceptions existent mais jouent un rôle mineur dans l'objet de notre étude.

Remarque 4: En observant les exemples précédents on s'aperçoit que, à ké et à té ké correspondent les mêmes stratégies distributionnelles :

28. Ké ja (futur); té ké ja (conditionnel)

29. Ké ka (futur); té ké ka (conditionnel)

30. Ké ja ka (futur); té ké ja ka (conditionnel)

\section{Genèse des modalités du futur et du conditionnel dans les créoles à base lexicale française des Petites Antilles : le cas du guadeloupéen}

\subsection{De la perte de tous les paradigmes grammaticaux du verbe et de l'adjonction d'adverbes de temps}

Avec la prudente réserve qu'observe le linguiste face aux corpus écrits et anciens d'une langue, nous reconnaissons que des chroniqueurs des XVIIe et XVIIIe siècles des Petites Antilles, Bouton (1640), Pelleprat (1656), Chévillard (1659), Mongin (1672), cités entre autres dans Prudent (1980), G. Hazaël-Massieux (1996), M.-C. Hazaël-Massieux (2008), offrent des corpus permettant de faire des hypothèses plausibles sur l'aube de l'expression des temps, modes et aspects, en CBLF. Ces pères missionnaires affirment unanimement, en des termes différents, que les «Noirs » sur les habitations parlaient par l'infinitif des verbes, indiquant le temps par adjonction d'adverbes de temps : Hier, aujourd'hui, demain.

a) Pour comprendre la formation de la modalité du futur en créole guadeloupéen, on doit conceptualiser ce premier stade, même fugitif et théorique où, comme le laissent entrevoir les archives des missionnaires, c'est un adverbe de temps, demain, qui exprime le futur dans les pré-créoles sur l'habitation. C'est le degré 1 de l'expression temporelle de tout énoncé entre deux personnes où deux groupes sociaux n'ayant pas la même langue maternelle. 
b) En deuxième lieu vient la créolisation linguistique du XVIIIe siècle : le verbe aller sous sa forme $v a$, a été la première conceptualisé du futur. La créolistique émet l'idée qu'à cette période, il se produit une perte de tous les paradigmes grammaticaux, les prédicats verbaux en particulier prenant une forme invariable proche de l'infinitif $\mathrm{du}$ français (comme dans le texte de Pelleprat) ou de la deuxième personne du pluriel encore chez Pelleprat lui-même. Ici, les deux formes verbales : voulé (rappelant la forme actuelle vlé) et savé : toi savé que moi voulé....même si ce corpus peut être une caricature et donc est peu fiable.

c) Les deux stades précédents, même concomitants ou presque, sont antérieurs en théorie, à l'organisation du système TMA des créoles tels que nous les connaissons aujourd'hui, avec des marques TMA, té, ké... Du point de vue de l'analyse énonciative, cela ouvre un champ de possibles fort intéressants pour la grammaire du créole mais également pour une réflexion en didactique des langues : Soit l'énoncé suivant : an kontan (je suis content), lorsque l'énonciateur y ajoute ké : an ké kontan (je serai content); ou té ké, ou encore lorsqu'il y introduit la forme négative : an pé ké kontan, on peut formuler l'analyse selon laquelle cet énonciateur fait un choix, celui de la mise en relation par l'intermédiaire de ké ou té ké ou pé ké, entre le sujet grammatical et le noyau prédicatif. Il y a non seulement mise en relation, mais encore modification de la mise en relation par l'énonciateur. Du point de vue de la grammaire énonciative en créole, le temps, la modalité et l'aspect semblent dictés par le locuteur (Facthum-Sainton, 1997 : 37). Ce choix fait par le locuteur affecte la relation entre le sujet grammatical et le noyau prédicatif (Damoiseau, $2012: 87$ ).

\subsection{De l'évolution de l'expression de la modalité du futur simple en Guadeloupe du XVIIIème siècle à nos jours : une incursion dans la diachronie des TMA}

\subsubsection{L'unité lexicale «va» comme première expression du futur au XVIIIe siècle en CBLF de la Caraïbe}

Dès les premiers textes écrits en créole au XVIIIe siècle, en Haïti, en Guadeloupe et en Martinique, la forme consacrée à la modalité du futur, à la forme affirmative, est incontestablement $v a$ parfois $a$. La proclamation $d u$ Cap, le 11 juillet 1793, texte publié par les autorités lors des événements politiques autour de l'indépendance d'Haïti, atteste cette unité lexicale, $v a$, en abondance ${ }^{5}$. La proclamation de Delpech, Haïti, le 13 juillet 1793 ne démentit pas cet usage abondant de va. Le texte ancien, biblique, la passion de notre Seigneur selon Saint-Jean en Langage Nègre, reproduit dans G. Hazaël-Massieux (1996) et M.-C. Hazaël-Massieux (2008) qui selon les points de vue courants s'inspire des pratiques langagières créoles des trois pays au XVIIIe siècle en est un exemple également. Exemples entre autres dans ce texte : c'est li meme qui va trahi moé (Hazaël-Massieux, $2008: 63$ ). Et un peu plus loin (p. 63-64) : [...] mais bientôt moé va lévé et moé va macé devant montré zottes chimin galilée ${ }^{7}$.

\footnotetext{
${ }^{5}$ L'article IV de cette déclaration en est un exemple : Nion semaine assez après que déclaration là va lire devant toute monde, à que li va affichée, comme nous sorti dire là, mariage là va faire (Hazaël-Massieux, 2008 : 201). ${ }^{6}$ [...] c'est li meme qui va trahi moé »...c'est celui-là même qui va me trahir ». (Extrait de l'Evangile selon St Jean en langage Nègre).

${ }^{7}$ [...] mais bientôt moé va lévé et moé va macé devant montré zottes chimin galilée » mais bientôt, je me leverai et je vais marcher devant, afin de vous montrer le chemin de la Galilée ».
} 
Maint locuteur du haïtien, aujourd'hui encore, continue à utiliser va. En Guadeloupe, en Martinique, cet élément lexical a progressivement disparu.

\subsubsection{La forme «sra»}

Hazaël-Massieux note que cette forme a été rare en Guadeloupe et en Martinique. Nous l'identifions dans les Petites Antilles entre 1822 (chez Félix Longin signalé dans son Voyage à la Guadeloupe, $2008: 431^{8}$ ) et $1848^{9}$ (Sang Froid des Schoelcheristes).

\subsubsection{La forme "ké», en compétition avec la forme "va», au milieu du XIXe siècle pour la modalité du futur}

En effet, à se baser sur les corpus écrits du créole de la moitié du XIXe siècle, on peut formuler l'hypothèse selon laquelle la forme ké et la forme va coexisteront pendant un certain temps. C'est le cas chez Marbot (Martinique), comme le note Hazaël-Massieux (2008 : 432433), par exemple dans « la laitière et le pot au lait en créole martiniquais ». En Guadeloupe la forme $v a$ semble être en nette régression par la rareté des emplois dont témoignent les textes au tournant du siècle, 1849 ${ }^{10}$. Il est rapporté (Hazaël-Massieux, $2008:$ 435) que la forme ké prédomine dans les textes polémiques des agriculteurs dans la presse écrite de Basse-Terre à partir de 1849. Ké du futur est attesté plus d'une dizaine de fois dans un court texte de polémique politique, alors que va n'est pas du tout attesté dans ce texte (Dakoé, Basse-Terre Guadeloupe, juillet 1949). ${ }^{11}$

\subsubsection{L'unité lexicale «pou»}

L'unité lexicale prépositionnelle pou fait partie de la modalité du futur. Son étymologie remonte à la préposition pour du français. Cette préposition en créole a évolué vers diverses étapes de métaphorisation, à savoir l'intention, la prédiction, la prévision la destination, l'objectif, le but :

31. I pou rivé a katrè (Il est prévu qu'il/elle arrive à quatre heures).

Il s'allie à té placé devant lui, pour une prévision future dans le passé par rapport au moment de l'énoncé : $i$ té pou rivé a katrè (il était prévu qu'il/elle arrive à quatre heures).

La même métaphorisation a été réalisée avec l'unité lexicale $f u$, [fu] des créoles à base lexicale anglaise, étymologie for, de l'anglais.

Malgré son apparition plutôt tardive dans la formation des CBLF des Petites Antilles, la marque TMA ké témoigne d'un ancrage dans les textes du milieu du XIXe siècle ${ }^{12}$, en Guadeloupe.

\footnotetext{
${ }^{8}$ Longin : Mon sra dit vous « je vous dirai » (Hazaël-Massieux, $2008: 431$ ).

${ }^{9}$ Idylles dans Hazaël-Massieux (2008: 432) : mon sra rende vous avant récolte (La cigale et la Fourmi) : «Je vous le rendrai avant la récolte ».

${ }^{10}$ Li va badiner cé la ki sottes, mais pas moin (Joseph Ibo, 3 juillet 1849 Dans Hazaël-Massieux, 2008 : 319). Le 11 juillet 1849 du même auteur, p. 320 : ça ki save palé va palé «ceux qui savent parler, parleront».

${ }^{11}$ [...] d'aillè can pays ké en haziers, toutes moune ké pauves, ces gras sicriers la ké obligé péché tactaques pou yo mangé, alos toutes moune ké égal (Hazaël-Massieux, 2008 : 301).

${ }^{12}$ Ké devant un verbe : Fleu zimmôtelle...li ké fè toué rété toujous kon zîmmôtelle (Baudot, $1980: 8$ ). «La fleur d'immortelle...elle te fera rester toujours immortelle ».

Ké devant l'auxiliaire tini : Pou zòtes, ké tini la grande citée chérie (Baudot, 1980 : 10).
} 


\subsection{L'évolution de la forme du conditionnel dans l'histoire du créole guadeloupéen}

La forme contemporaine, té ké, ne semble pas attestée avant le XIXe siècle. Selon toute vraisemblance, cette forme a été précédée de la forme $s e^{13}$ avec laquelle elle a cohabité pendant un certain temps. Puis sré va disparaître en Guadeloupe, le point de départ de sa fluctuation se situant autour des années 1848. Sré n'est pas sans rappeler la forme actuelle martiniquaise, sé. Comme dit précédemment, au XIXe siècle, ké et té ké/té qué correspondent à un phénomène émergeant, mais té occupe une place fondatrice dans toute la littérature écrite depuis les premiers écrits en créole du XVIIIe siècle, soit seul soit avec ka/ca/qua/qu'à.

Les textes créoles produits en Guadeloupe au milieu du XIXe siècle, ceux des scriptes célèbres, Pengoué, Ibo, Dakoé, attestent par la graphie, que la forme té ké n'est pas encore complètement intégrée dans les habitudes des locuteurs. En effet, certains scriptes de l'époque semblent ne pas distinguer au plan graphique, pa té et pa té ké, comme vraisemblablement $p a$ té ké se prononce également [pa te :] avec une voyelle longue dans [te :], suite à l'effacement de la consonne sourde vélaire [k] de $k e^{14}$. Cette erreur de graphie nous semble être également une erreur de prononciation. C'est d'ailleurs la même erreur que commettent ceux des locuteurs non natifs apprenant le créole.

Par ailleurs, une variation graphique té ké/té qué est attestée chez un même scripte, Dakoè, personnage connu de la littérature créole ancienne ${ }^{15}$.

On notera qu'au XIXe siècle, la forme faudré, forme impersonnelle du modal falloir au conditionnel (il faudrait que) est utilisée, par exemple chez Baudot, textes en créole guadeloupéen $^{16}$. Aujourd'hui, cette forme est obsolète en Guadeloupe, alors qu'elle est en usage en Martinique.

Ké devant un groupe prépositionnel introduit par une préposition de lieu: d'aillè can pays ké en haziers «D'ailleurs, quand le pays sera mal portant » (Dakoè dans Hazaël-Massieux, 2008).

Ké devant un adjectif : toutes moune ké pauves (Dakoè dans Hazaël-Massieux, 2008).

Dans ces exemples, n'est pas attesté l'usage identifié de Ké devant un substantif, comme en Guadeloupe actuellement : $i$ ké nonm, kon ou ka vwè’y la « Tel qu'il est maintenant, il fera un maître homme »

${ }^{13}$ Dans Fondoc et Thérèse (Baudot, opéra en un acte remontant à 1856) atteste cette forme de manière continue : Si moin té vlé dentelle, si moin té vlé l'agent, moins sré trapé tout ça (1935 : 167) «Si je voulais de la dentelle, si je voulais de l'argent, j'aurais tout cela ».

Dans «Pour le temps de Noël » quelques années avant Baudot, on trouve une alternance graphique c'rélcé : Nous plus fort pacé cé gens-la, nou s'ré fè yo dansé polka (Hazaël-Massieux, 2008 : 277- Ca nous cé fè si nous té nommé li (Hazaël-Massieux, 2008 : 286) « Nous sommes plus fort que ces gens-là, nous leur ferions danser la polka (si nous le voulions) ».

Joseph Ibo, 1849 : si blancs té payé, nous s'ré vouès ca (Hazaël-Massieux, 2008 : 320) « si les blancs avaient payé, nous nous en rendrions compte ».

${ }^{14}$ Dans l'énonce, sans li, mouché libèté pas té changé agnen, il aurait fallu avoir non pas, pa té, mais pa té ké. C'est encore Dakoè qui ne distingue pas té de té ké à la forme affirmative 7 juillet 1849: Si bon Dié té méchant, tini longtemps tonnè té crasé nègue la (Hazaël-Massieux, 2008: 301), au lieu de té ké crasé nègue la. L'impression que peut avoir le linguiste est la précarité des formes té ké, et pa té ké, comme une phase de transition de sré à té ké après l'abolition de l'esclavage.

${ }^{15}$ Dakoè, 6 décembre 1849 : Si zote té ca chassé blanc, Répiblique té ké voyé soda pou fè zòt maron «Si vous chassiez les blancs, la République enverrait des soldats pour vous faire fuir ».

Dakoè, quelques mois avant, septembre 1849 : Mon té di ou mon pa té qué écrit vous encore «Je vous avais dit que je ne vous écrirais plus ».

${ }^{16}$ Baudot (1980 : 28) : faudré gnon jou entiè pou raconté tou ça «il faudrait un jour entier pour raconter tout cela ». Les exemples nous semblent légions chez Baudot (1856:164) : faudré mwen sré bien bête, tracassé mwen la tête,... «Il faudrait que je sois bien bête pour me tracasser la tête ». Aujourd'hui, c'est une forme bien 


\section{La contribution de la grammaire du créole à la contextualisation didactique}

\subsection{Constats sur le terrain de l'école en Guadeloupe : la difficulté de modaliser correctement en français}

Dans les échanges constants que nous avons avec des enseignants en Guadeloupe, ces derniers sont de plus en plus nombreux à affirmer empiriquement que, face aux sérieuses difficultés des élèves par rapport aux notions de temps en français, ils n'ont aucun recours didactique officiel. Par contre, une simple allusion aux TMA du créole, disent ces enseignants, se révèle être éclairante pour l'élève. Donc, l'usage et les notions impliquées dans la combinaison té ké ja ka, en dépit de leur complexité, semblent être une référence sûre pour l'élève, car elle est mieux intériorisée que la stratégie flexionnelle française.

Pour comprendre le problème, Olivia Boudhau, étudiante de master 2 en professorat des écoles à l'ESPE de l'académie de la Guadeloupe, dans le cadre de son mémoire au sein du parcours «Langue et Culture Régionales », a ébauché une expérimentation qui n’a pas encore abouti mais qui donne à réfléchir. Elle a fait passer un test d'évaluation diagnostique à 12 élèves d'une classe de CM1, et à 17 élèves d'une autre classe de CM1, cette dernière étant une classe bilingue créole-français. L'exercice consistait à compléter à sa guise le début de phrase : Si j'avais de l'argent... Les élèves n'avaient pas encore étudié le conditionnel. Voici les résultats obtenus :

- Si j'avais de l'argent, j'aurais acheté une voiture (Réponse de 11 élèves sur 12 en CM1 non bilingue ; Réponse de 13 élèves sur 17 en CM1 bilingue) ;

- Si j'avais de l'argent, j'allais acheter une voiture (Réponse de 3 élèves en CM1 bilingue) ;

- Si j'avais de l'argent, j'irai acheter une voiture (Réponse de 1 élève sur 12 en CM1 non bilingue).

Par ailleurs, pour savoir si le milieu créole était la cause de ces énoncés erronés au sein de ces deux classes de CM1, la stagiaire a fait réagir deux élèves nés en France métropolitaine et de souche métropolitaine et un autre élève de CE1 remplissant les mêmes conditions d'origine que les deux autres : elle a obtenu les réponses suivantes, sans la moindre hésitation affirme-telle :

- Léana (élève a de CE1): Si j’avais de l'argent, je ferais un métier dans un restaurant ;

- Elève b de CM1 : Si j'avais de l'argent, j'achèterais une belle robe ;

- Autre élève c de CM1 en Guadeloupe depuis 4 ans: Si j'avais de l'argent, je déménagerais et je m'achèterais un bateau.

Autrement dit, dans les deux classes, tous les énoncés construits par les élèves natifs, et comportant la modalité du conditionnel, se révèlent faux quant à l'application de la concordance des temps. En revanche, même s'ils évoluent en contexte créole, les quelques élèves métropolitains interrogés, maîtrisent la règle normée qui consiste à faire usage de l'imparfait dans la subordonnée de condition et le conditionnel présent dans la proposition principale. 
La problématique première qu'on pourrait formuler est de savoir si ces chiffres sont représentatifs des compétences linguistiques de la majorité des élèves natifs de Guadeloupe en CM1. L'hypothèse déjà formulée est que, étant donné la compétence linguistique des 3 petits métropolitains à bien gérer la concordance des temps au conditionnel, le créole ne serait-il pas source de ces difficultés des natifs de Guadeloupe, en français?

Olivia Boudhau a par ailleurs décidé de faire passer un test d'évaluation diagnostique, en créole, à tous les enfants qui ont participé au premier test. Ce test consistait à compléter l'énoncé suivant : si an té ni lajan... Ses résultats présentent $97 \%$ de réussite, dit-elle. Elle en conclut que si les élèves maîtrisent si bien le conditionnel en créole, il serait intéressant d'utiliser la grammaire du créole pour faire émerger le déjà-là et pour ensuite faire le transfert des compétences en français. La question qui se pose est de savoir si cet échantillon de bonne compétence linguistique de ces élèves en particulier, à propos du conditionnel en créole, est représentatif du niveau de grammaire créole de tous les élèves natifs de Guadeloupe.

Face à ces hypothèses et problématiques dont nous n'aurons pas la réponse dans la présente étude, il ne s'agira pas, à cette étape de notre travail, d'élaborer une démarche d'enseignement du conditionnel en créole pour des élèves créolophones. Il s'agira surtout de donner à l'enseignant, souvent démuni, le matériel grammatical théorique et pratique nécessaire pour la maitrise du futur et du conditionnel, afin qu'il soit autonome dans sa classe de LVR. Cidessous, nous proposons de mettre en contraste le créole et le français dans la règle de la concordance des temps, selon les trois concepts que nous avons appelés ci-dessous, l'éventuel, le potentiel et l'irréel, espérant ne pas perdre l'enseignant dans la terminologie qu'il emploie habituellement ${ }^{17}$.

\subsection{Le contraste créole-français comme support pour l'enseignant dans sa démarche de sensibilisation de ses élèves à la grammaire du créole}

Chez certains locuteurs guadeloupéens, exprimer correctement l'équivalent français de la phrase créole ci-dessus se heurte à la difficulté de la concordance des temps, concordance des temps qui ne se manifeste pas de la même façon en créole :

\begin{tabular}{|l|l|l|}
\hline \multicolumn{1}{|c|}{ Modalités } & \multicolumn{1}{c|}{ Exemples en créole } & \multicolumn{1}{c|}{ Traduction en français } \\
\hline $\begin{array}{l}\text { 1. L'ÉVENTUEL } \\
\text { (Eventualité plus probable } \\
\text { que dans le potentiel) }\end{array}$ & Si ou (ka) travay, ou ké rivé & Si tu travailles, tu réussiras \\
\hline $\begin{array}{l}\text { 2. LE POTENTIEL } \\
\text { (Hypothétique de la fiction } \\
\text { possible) }\end{array}$ & $\begin{array}{l}\text { Si ou té ka travay, ou té kéé } \\
\text { rivé }\end{array}$ & Si tu travaillais, tu réussirais \\
\hline $\begin{array}{l}\text { 3. L'IRRÉEL } \\
\text { (Hypothétique de la fiction } \\
\text { démentie par les faits) }\end{array}$ & Si ou té travay, ou té ké rivé & $\begin{array}{l}\text { Si tu avais travaillé, tu aurais } \\
\text { réussi }\end{array}$ \\
\hline
\end{tabular}

Figure 1 : Observation de la concordance des temps en créole et en français dans des phrases impliquant une proposition subordonnée de condition

\footnotetext{
${ }^{17}$ Les analyses de la modalité du conditionnel sont très variées. Là où nous avons utilisé le terme « le potentiel », correspond parfois, la terminologie «irréel du présent» et là où il y a «l'irréel » dans notre tableau, cela correspond chez d'autres auteurs à « irréel du passé ».
} 


\subsection{La règle de la concordance des temps en créole peut-elle contribuer à la prise de conscience de la règle de concordance des temps dans l'apprentissage du français et des autres langues de l'école ?}

Une analyse du tableau ci-dessus montre qu'il est possible de s'appuyer jusqu'à un certain point sur la concordance des temps en créole pour comprendre la notion générale de concordance des temps dans les langues du monde. En effet, futur et conditionnel peuvent être mis en parallèle, dans l'éventuel et le potentiel :

Lorsque la proposition subordonnée de condition est au présent optatif (si ou ( $k a$ ) travay), la proposition principale est au futur (ou ké réyisi).

Mais lorsque la proposition subordonnée de condition est à l'imparfait ou ce qui correspond à l'imparfait en créole (té $k a$ ), le verbe de la proposition principale est au conditionnel (té ké).

Ceci correspond à des usages similaires en français, en anglais, en espagnol.

L'enseignant peut s'appuyer sur ce point essentiel de la concordance des temps en créole.

L'enseignant peut par ailleurs s'appuyer sur la logique du raisonnement menant à la concordance des temps, à partir du créole pour mieux comprendre les temps utilisés: il s'agit de cette distinction en créole entre l'éventuel, le potentiel et l'irréel :

a) Dans l'éventuel, le procès est plus probablement réalisable que dans le potentiel. Le raisonnement dans l'éventuel ramène l'enseignant à cette logique qui correspond à une attitude culturelle des peuples, populaire en Guadeloupe : travay, é ou ké rivé, pa ni pwoblenm ${ }^{18}$. L'enseignant peut utiliser cette glose (populaire) pour que l'élève ressente l'éventuel.

b) Le potentiel exprime comme le disent certains, un hypothétique de la fiction possible, mais déjà moins probable que l'éventuel : (kon-yé-la, ou ni tan ankò pou réyisi, men ou pa ka fè ayen pou sa ${ }^{19}$ ). L'enseignant peut aussi utiliser cette glose, familière dans les usages créoles, pour expliquer le potentiel.

c) L'irréel dont la fiction est démentie par les faits (Ou pa travay ni yè, ni avan-yè, ki fè, ayen pé pa fèt ankò ${ }^{20}$ ) correspond à la manière dont la mère va gronder l'enfant devant son échec. C'est aussi une forme qui peut servir de glose.

\subsection{S'appuyer sur les divergences créole-français, pour comprendre l'échec des élèves créolophones dans le principe de la concordance des temps : potentiel/irréel}

L'enseignant doit être particulièrement vigilant dans l'observation des propositions principales du potentiel et de l'irréel :

\footnotetext{
${ }^{18}$ Travay, é ou ké rivé, pa ni pwoblenm ${ }^{18}$ (Travaille et tu réussiras ; il n'y a aucune difficulté).

${ }^{19}$ Kon-yé-la, ou ni tan réyisi ankò, men ou pa ka fè ayen pou sa (A cette étape, tu as encore le temps de réussir, mais tu ne fais aucun effort en ce sens)

${ }^{20}$ Men ou pa travay ni yè, ni avan-yè, ki fè ayen pé pa fèt ankò (Mais, tu n'as travaillé ni hier, ni avant-hier, alors plus rien n'est possible).
} 


\begin{tabular}{|l|l|l|}
\hline \multicolumn{1}{|c|}{ Modalités } & \multicolumn{1}{|c|}{ Exemples en créole } & \multicolumn{1}{c|}{ Traduction en français } \\
\hline Le potentiel & $\begin{array}{l}\text { Si ou té ka travay, ou té ké } \\
\text { rivé }\end{array}$ & Si tu travaillais, tu réussirais \\
\hline L'irréel & Si ou té travay, ou té ké rivé & $\begin{array}{l}\text { Si tu avais travaillé, tu aurais } \\
\text { réussi }\end{array}$ \\
\hline
\end{tabular}

Figure 2 : Le contraste créole-français dans la proposition principale et la proposition subordonnée de condition, au sein d'énoncés mettant en vis-à-vis le potentiel et l'irréel

Dans la proposition principale, le créole neutralise l'opposition dite «conditionnel présent/conditionnel passé ». C'est la seule forme té ké (Temps-Mode) qui est utilisée dans les deux phrases en créole que ce soit pour l'expression du potentiel ou de l'irréel.

En revanche, en français, je réussirais et j'aurais réussi, dans la proposition principale, expriment la distinction entre un conditionnel présent et un conditionnel passé.

Etant donnée cette neutralisation de l'opposition passé/présent dans la proposition principale en créole, on ne trouvera pas étonnant que soient attestés en Guadeloupe les énoncés modaux erronés tels ceux des élèves des deux classes que notre stagiaire a eu à conduire.

On pourra cependant en créole, s'appuyer sur l'usage de la forme té, passé antérieur, dans la proposition subordonnée de condition introduite par «si », pour faire voir la différence entre le potentiel et l'irréel en créole: si ou té ka et si ou té. Dans le cas de l'irréel, le marqueur té employé seul met fin à toute expectation.

\subsection{L'expression du futur « simple » : mise en contraste du créole et du français sur les bases de la réalité des enseignements dispensés à l'école}

Nous travaillons sur la base de cet enseignement dispensé à l'école, celui du futur simple dans les deux langues et non pas simplement du futur. Le tableau ci-dessous peut, à juste titre, être considéré comme faussé, dans la mesure où comme le dit Chaudenson dans son avant-propos aux guides du maître (2010), la forme française à laquelle la forme créole devrait être mise en contraste est la périphrase «verbe aller+infinitif) : je vais parler/An ké palé.

\begin{tabular}{|l|l|}
\hline \multicolumn{1}{|c|}{ Créole } & \multicolumn{1}{|c|}{ Français } \\
\hline An ké palé & Je parlerai \\
\hline Ou ké palé & Tu parleras \\
\hline I ké palé & Il/elle parlera \\
\hline Nou ké palé & Nous parlerons \\
\hline Zòt ké palé & Vous parlerez \\
\hline Yo ké palé & Ils/elles parleront \\
\hline
\end{tabular}

Figure 3 : Stratégies de deux expressions du futur en créole et en français

Le Futur simple : quelles convergences et quelles divergences entre créole et français?

Convergences:

- Le créole et le français ont en commun qu'il existe dans les deux langues des stratégies propres pour marquer le futur simple. C'est là une convergence sur laquelle on peut fonder une hypothèse de démarche pour faire émerger le déjà-là et la 
confiance de l'élève sur cette notion du futur qui au fond ne lui est pas aussi étrangère qu'elle le paraît en situation d'apprentissage.

\section{Divergences :}

- La grammaire créole exprime la temporalité par des marqueurs préverbaux placés devant le verbe : la notion du futur s'exprime souvent par le marqueur préverbal ké, antéposé au verbe. En français en revanche, il s'agit d'une désinence verbale, -ai, -as, $-a$, -ons, $-e z$, -ont.

- En français, la désinence du futur est soumise à l'appartenance du verbe à un groupe dont l'infinitif se termine respectivement par -er, -ir, et pour le troisième groupe, -re, $i r$, -oir, -oire, ce qui rend variée et plus complexe la forme du futur: chanterai, finirai, prendrai, boirai. Il n'en est rien pour le créole dont la forme du verbe et du marqueur TMA ké sont invariables.

- Contrairement au créole, en français, la désinence verbale est soumise à la catégorie de la personne : je chanterai, tu chanteras versus an ké chanté, ou ké chanté.

\section{Conclusion}

L'étude de la modalité du futur et du conditionnel en créole guadeloupéen, à des enseignants guadeloupéens, donc créolophones, quelle que soit leur compétence en créole, nous a permis d'aller assez loin, dans l'analyse énonciative, en synchronie, en diachronie et dans la démarche contrastive, parce que le public auquel s'adresse le cours est créolophone et n'est pas sans connaissance sur les questions que nous avons traitées. Nous espérons que cette réflexion en contextualisation didactique de la modalité du futur et du conditionnel aura posé les jalons pour une meilleure approche de la grammaire par le biais du créole. Nous nous excusons d'avance des points faibles de notre analyse didactique, l'initiative n'étant qu'à ses débuts. Nous souhaitons que certaines des connaissances rendues disponibles dans cette approche soient transposables aux élèves, avec toutes les faiblesses de notre analyse à l'étape actuelle.

\section{Références bibliographiques}

Alleyne, M. (1996). Syntaxe historique créole. Paris : Karthala.

Arends, J., Muysken, P. et Smith, N. (dir.) (1995). Pidgins and Creoles : An introduction. Philadelphie : John Creole language library, Benjamins Publishing Company.

Baudot, P. (1980). Oeuvres créoles. Basse-Terre: Comité départemental de l'Année du patrimoine.

Bernabé, J. (1983). Fondal-natal: grammaire basilectale approchée des créoles guadeloupéen et martiniquais. Paris : L'Harmattan.

Bernabé, J. (1987). Grammaire Créolelfondas kréyòl-la. Paris : L'Harmattan.

Bernabé, J. (2000). Précis de syntaxe créole, série Guide de langues et de Cultures créoles. Cayenne : Ibis Rouge.

Bickerton, D. (1981). Roots of language. Karoma : Ann Arbor, Michigan

Bickerton, D. (1984). The language bioprogram Hypothesis. Behavioral and Brain Sciences, 7(2), 173-203.

Bickerton, D. (1988). Creole languages and the bioprogram. Dans F. Newmeyer (dir.) Linguistics : The Cambridge Survey. Linguistic theory : Extensions and Implications (p. 268-284). Cambridge : Cambrige University Press.

Chaudenson, R. (1992). Des Iles, des Hommes, des Langues. Paris : L'Harmattan.

Creissels, D. (2004). Cours de syntaxe générale, chap. 10. Non publié. 
Damoiseau, R. (2012). Syntaxe créole comparée, Martinique, Guadeloupe, Guyane, Haïti. Paris : Editions Karthala et CNDP-CRDP.

Damoiseau, R. (1999). Eléments de grammaire comparée français-créole martiniquais. Guyane : Ibis Rouge et Presses Universitaires créoles/GEREC-F.

Damoiseau, R. (1984). Eléments de grammaire du créole martiniquais. Fort-de-France: Hatier.

DeGraff, M. (1992). The syntax of Predication in Haitian. Dans K. Broderick (dir.) Proceedings of NELS 22 (p. 103-117). Amherst, Mass : Graduate Linguistics Student Association.

DeGraff, M (2004). Word order and morphology in «creolization » and beyond. Dans G. Cinque and R. Kayne, Handbook of comparative syntax (p. 249-312). New York: Oxford University Press.

Durizot Jno-Baptiste, P. (1996). La question du créole à l'école en Guadeloupe. Paris : L'Harmattan.

Facthum-Sainton, J. (1997). Té ké ja ka, une perception de l'espace et du temps en créole : l'exemple du créole guadeloupéen. Dérades revue caribéenne de recherches et d'échanges, 1, 35-47.

Fattier D. (2010). L'acquisition non guidée de langue étrangère (ALE) comme facteur de la créolisation linguistique : l'exemple des marqueurs AP et KA. Etudes créoles, 1, 111123.

Hazaël-Massieux, G. (1996). Les créoles. Problèmes de genèse et de description. Aix-enProvence : Publication de l'université de Provence.

Hazaël-Massieux, G. (1993). L'expression du futur en créole mauricien. Etudes créoles, 16(1), 61-75.

Hazaël-Massieux, G. (1992). Genèse des marques de TMA en créole de Guadeloupe. In G. Hazaël-Massieux (dir.), Les créoles. Problèmes de genèse et de description (p. 221248). Aix-en-Provence : Publication de l'université de Provence.

Hazaël-Massieux, G. (1991). Genèse ou histoire de la modalité verbale en créole de Guadeloupe. Dans G. Hazaël-Massieux (dir.), Les créoles. Problèmes de genèse et de description (p. 17-30). Aix-en-Provence : Publication de l'université de Provence.

Hazaël-Massieux, G. (1986). La créolisation est-elle un phénomène limité dans le temps ? L'expression du futur en Guadeloupe. Etudes créoles, 9(1), 114-126.

Hazaël-Massieux, M.-C. (2008). Textes anciens en créole français de la Caraübe. Histoire et analyse. Paris : Publibook.

Howe, K. (2000). Développement d'une théorie de la temporalité : les cas du Créole Haïtien et du Papiamento. Thèse de doctorat non publiée. Aix-en-Provence: Université de Provence,

Jeannot-Fourcaud, B. (2003). De l'opposition verbo-nominale en créole martiniquais. Approche fonctionnaliste. Thèse de doctorat non publiée. Paris: Université Paris Descartes.

Kriegel, S. et Arends, J. (2003). Grammaticalisation et réanalyse : approches de la variation créole et française. Paris : CNRS.

Lapaire, J.-R. et Rotgé, W. (1991). Linguistique et grammaire de l'anglais. Toulouse : Presses universitaire du Mirail.

Marchello-Nizia, C.(2001). Grammaticalisation et évolution des systèmes grammaticaux. Langue Française, 130, 31-44.

Mufwene, S. (1985). Observation on Time Reference in Jamaican and Guyanese Creolesdans Studies in Caribbean Language. Trinidad et Tobago: Society for Caribbean Linguists. 
Mufwene, S. (2001). The ecology of language evolution. Cambridge : Cambridge University Press.

Prudent, L.-F. (1980). Des baragouins à la langue antillaise. Paris : Editions Caribéennes.

Rotgé, W. et Lapaire, J.-R. (2004) : Réussir le commentaire grammatical de Textes. CapesAgreg anglais. Ellipses.

Rotgé, W. et Lapaire, J.-R. (2014). Linguistique et grammaire de l'anglais, 3 édition, PUM.

Spears, A. (1997). Vers un modèle des systèmes Temps Mode Aspect dans trois langues créoles : l'Haïtien, le Guadeloupéen et le Martiniquais. Etudes créoles, 20(2), 85-91.

Valdman, A. (1978). Le créole : Structure, Statut et Origine. Paris : Klincksieck. 